\title{
Early-onset GH deficiency results in spatial memory impairment in mid-life and is prevented by $\mathrm{GH}$ supplementation
}

\author{
E Nieves-Martinez ${ }^{1, *}$, W E Sonntag ${ }^{5, *}$, A Wilson ${ }^{2}$, A Donahue ${ }^{2}$, D P Molina ${ }^{3}$, \\ J Brunso-Bechtold ${ }^{1,3}$ and $M$ M Nicolle ${ }^{1,2,4}$ \\ ${ }^{1}$ Neuroscience Program, ${ }^{2}$ Physiology and Pharmacology, ${ }^{3}$ Neurobiology and Anatomy and ${ }^{4}$ Department of Internal Medicine, Section of Gerontology, Wake \\ Forest University Health Sciences, Medical Center Boulevard, Winston-Salem, North Carolina 27157-1083, USA \\ ${ }^{5}$ Reynolds Oklahoma Center on Aging, Oklahoma University Health Sciences Center, Oklahoma City, Oklahoma 73104, USA \\ (Correspondence should be addressed to M M Nicolle; Email: mnicolle@wfubmc.edu) \\ *(E Nieves-Martinez and W E Sonntag contributed equally to this work)
}

\begin{abstract}
$\mathrm{GH}$ levels increase to high concentrations immediately before puberty then progressively decline with age. GH deficiency (GHD) originating in childhood is treated with $\mathrm{GH}$ supplementation to foster somatic development during adolescence. It is not clear if or how early GH replacement affects memory in adulthood, or whether it can prevent the cognitive deficits commonly observed in adults with childhood-onset GHD. Rats homozygous for the Dw-4 mutation (dwarf) do not exhibit the normal increase in $\mathrm{GH}$ at 4 weeks of age when $\mathrm{GH}$ levels normally rise and are used to model childhood or early-onset GHD (EOGHD). One group of these rats was injected with $\mathrm{GH}$ from 4 to 14 weeks of age to model GH supplementation during adolescence with GHD beginning
\end{abstract}

in adulthood (adult-onset GHD; AOGHD). Another group received $\mathrm{GH}$ from 4 weeks throughout the lifespan to model normal lifespan GH (GH-replete). Age-matched, Dw-4 heterozygous rats (HZ) do not express the dwarf phenotype and were used as controls. At 8 and 18 months of age, spatial learning in the water maze was assessed. At 8 months of age all experimental groups were equally proficient. However, at 18 months of age, the EOGHD group had poor spatial learning compared to the AOGHD, GH-replete, and HZ groups. Our data indicate that GHD during adolescence has negative effects on learning and memory that emerge by middle-age unless prevented by $\mathrm{GH}$ supplementation.

Journal of Endocrinology (2010) 204, 31-36

\section{Introduction}

The levels of $\mathrm{GH}$ and its effector insulin-like growth factor 1 (IGF1) vary substantially throughout the lifespan (Nyberg 2000). Postnatal GH and IGF1 levels are low, but increase to higher concentrations immediately before puberty, and then progressively decline with increasing age. Deficits of $\mathrm{GH}$ in childhood can be due to a congenital deficiency or acquired in either childhood or adulthood due to hypothalamicpituitary tumors, $\mathrm{GH}$ insensitivity, or disorders involving $\mathrm{GH}$ secretion. The clinical emphasis on treating childhood-onset $\mathrm{GH}$ deficiency (COGHD) currently focuses on the somatic role of $\mathrm{GH}$ in increasing body size (mainly height) during puberty (Clayton et al. 2007, Nilsson et al. 2007). Continued GH treatment during this important transition period between adolescence and adulthood may be important for the normal development of bone and muscle in adulthood (20-30 years old) (reviewed in Clayton et al. (2007) and Nilsson et al. (2007)). As the body continues to develop into young adulthood in humans, the brain also continues to mature (Rice \& Barone 2000). The effects of COGHD on cognition in adulthood indicate that it causes impairments on measures of hippocampal/medial temporal lobe function. For example, a study of 24-year-old subjects with COGHD and age-matched controls indicated that COGHD results in poor performance on delayed verbal memory when compare to age-matched controls (van Dam et al. 2005). However, it is not clear if the adult-onset of this memory impairment can be remediated by $\mathrm{GH}$ supplementation earlier in the lifespan.

Dwarf (Dw-4/Dw-4) rats have chronically low levels of GH and IGF1 and make an excellent animal model of COGHD (Charlton et al. 1988). These rats have a spontaneous mutation that results in decreased $\mathrm{GH}$ secretion from the pituitary (Charlton et al. 1988, Carter et al. 2002a,b). A relatively circumscribed exposure of the dwarf rats to $\mathrm{GH}$ during postnatal weeks 4-14 (peripubertal to young adulthood life stages) influences the timing of intra-cerebral hemorrhage or cardiac thrombus later in life and increases the lifespan by $15 \%$ (Sonntag et al. 2005). This has led to the conclusion that manipulation of $\mathrm{GH}$ levels for a brief transitional period starting just around puberty and ending in young adulthood 
is sufficient to alter age-related pathology later in life. It is not known if this circumscribed exposure also influences cognition in adulthood and midlife.

The present study was designed to further explore the consequences of alterations in the peripubertal rise in $\mathrm{GH}$ on memory in adult animals. In the Lewis dwarf rats, puberty begins around postnatal day (PD) 35-36, just after the increase in GH pulsatility and the subsequent IGF1 increase, which begins around PD28. Brain development continues through adolescence to young adulthood (PD63-70) (McCutcheon \& Marinelli 2009). Because of the high density of GH and IGF1 receptors in the hippocampus (Adem et al. 1989, Lai et al. 1993, Sonntag et al. 1999) and the finding that IGF1 infusion can restore the age-related decline in hippocampal-dependent memory (Markowska et al. 1998), hippocampal-dependent memory was assessed using a standardized assessment of spatial learning in a water maze that has been demonstrated to be sensitive to the effects of age (Gallagher et al. 2003, LaSarge \& Nicolle 2009). For this study, we examined spatial learning at 8 and 18 months of age in dwarf animals treated with vehicle alone to model COGHD (early-onset GHD; EOGHD). The spatial learning ability of the EOGHD rats was then compared with dwarf rats treated from 4 to 14 weeks of age to model a GH intervention in the periadolescent stage (adult-onset GHD; AOGHD). Long-term $\mathrm{GH}$ replacement (GH-replete) throughout the lifespan of dwarf rats was used to model lifetime supplementation with GH. In addition to the manipulations of $\mathrm{GH}$ in the dwarf rats, littermates that were heterozygous (HZ) for the autosomal recessive $D w-4$ mutation (non-dwarf phenotype) were used as controls to model normal life-time levels of GH. Our results support the conclusion that GHD during the periadolescent transition period results in deficits in learning and memory that are manifest at midlife. Furthermore, GH supplementation during this period, but not beyond, is sufficient to prevent and/or reduce this early decline in learning and memory.

\section{Materials and Methods}

\section{Subjects and experimental design}

Subjects are male Lewis rats that are heterozygous or homozygous for the spontaneous autosomal recessive $D w-4$ mutation which causes a decrease in $\mathrm{GH}$ secretion from the pituitary gland (Charlton et al. 1988). Female heterozygous $(D w-4 /-)$ Lewis rats were bred with male homozygous Lewis dwarf rats $(D w-4 / D w-4)$ to generate heterozygous (Dw-4/-) offspring with a normal phenotype (HZ) or homozygous rats $(D w-4 / D w-4)$ with a dwarf phenotype (dwarf). Classification as HZ or dwarf was based on their body weight at 28 days of age. Beginning on day 30 , dwarf rats were divided into three experimental groups: 1) EOGHD given saline, 2) AOGHD with GH administered between 4 and 14 weeks of age, or 3) GH-replete with GH administered beginning at 4 weeks of age and continued throughout the lifespan. Saline or GH $(200 \mu \mathrm{g}$ recombinant porcine $\mathrm{GH}$, Alpharma, Victoria, Australia) was s.c. injected twice daily. The HZ rats were used as controls and given saline injections twice daily from 4 weeks of age to the end of life. Terminology and treatment groups are described in Table 1. Two experimental cohorts were studied, one with water maze testing at 8 months of age and another with testing at 18 months of age. The first cohort had the following number of subjects per groups: HZ, 9; EOGHD, 5; AOGHD, 10; and GH-replete, 10. The second cohort had the following number of subjects per group: HZ, 14; EOGHD, 5; AOGHD, 13; and GH-replete, 13. Rats had access to food and water ad libitum and were housed in pairs in the vivarium of the Department of Physiology and Pharmacology at Wake Forest University Medical School. Body weights were measured prior to spatial learning assessment. All studies were approved by the Wake Forest University Institutional Animal Care and Use Committee.

\section{Spatial learning assessment}

Rats were tested in a water maze at either 8 or 18 months of age based on a standard assessment of spatial learning ability (Gallagher et al. 1993). The maze consisted of a circular pool (diameter, $1.83 \mathrm{~m}$ and height, $0.58 \mathrm{~m}$ ) with an escape platform centered in one of the four maze quadrants. Rats were trained over 12 sessions (three trials/day) to locate an escape platform hidden just below the water's surface. There were two types of trials, training trials and probe trials. During a training trial, the animal was allowed to swim for $90 \mathrm{~s}$ to locate the platform. Every sixth trial consisted of a 30 s probe trial to assess the development of a spatially localized search for the escape platform. During the probe trial, the platform was made unavailable for escape. The accuracy of the search

Table 1 Rat experimental groups

Genetic background

\section{Group/model}

Heterozygous ( $\mathrm{HZ})$

Early-onset GH deficiency (EOGHD)

Adult-onset GH deficiency (AOGHD) $\mathrm{GH}$ replete
$D w-4 /-$

$D w-4 / D w-4$

$D w-4 / D w-4$

$D w-4 / D w-4$
Treatment: 14 weeks of age until sacrifice

Treatment: 4-14 weeks of age

Saline vehicle, twice daily Saline vehicle, twice daily

$\mathrm{GH}$, twice daily

$\mathrm{GH}$, twice daily
Saline vehicle, twice daily Saline vehicle, twice daily Saline vehicle, twice daily $\mathrm{GH}$, twice daily 
for the platform location was measured by the proximity to the location for $30 \mathrm{~s}$ with the subject's position sampled ten times/s and summed. There were four interpolated probe trials, the sum of which was used to generate a composite score. Lower proximity scores represent a more accurate search. Cue training to a visible platform occurred on the last day of training to test for sensorimotor and motivational factors independent of spatial learning. Cue training consisted of six sequential trials with randomized start and visible platform locations.

\section{Measurement of plasma IGF1}

Rats were anesthetized with isofluorane and plasma was obtained via tail bleed within 1 week of behavioral assessment. Total IGF1 levels in plasma tissue homogenates were determined using R\&D Systems Quantikine mouse IGF1 Immunoassay (MG100; Minneapolis, MN, USA) as previously described (Adams et al. 2009). Briefly, blood was collected into tubes with 38 units porcine heparin per milliliter, incubated on ice, and centrifuged. The plasma was collected and stored in aliquots at $-80{ }^{\circ} \mathrm{C}$. For IGF1 measurement, samples were thawed to room temperature, gently vortexed, diluted serially in kit calibrator diluent at 1:2091 final, and assayed according to the manufacturer's instructions. Results are reported as nanogram IGF1 per milliliter plasma.

\section{Statistical analysis}

Training trial (hidden platform) data from the water maze were analyzed using repeated-measures ANOVA with group as a factor. Significant interactions were followed with Fisher's protected least significant difference (PLSD). Other measures were analyzed using a one-way (group) ANOVA unless otherwise specified. Data were analyzed within a specific age group ( 8 or 18 months of age) and not compared across ages due to the design of the experiment.

\section{Results}

\section{Spatial learning}

Spatial learning ability was assessed using a standardized protocol in the water maze (Gallagher et al. 2003, LaSarge \& Nicolle 2009). At 8 months of age, all groups became more proficient with increased training during the acquisition phase of the water maze indicated by a main effect of training trial block $(F(3,4)=137 \cdot 81, P<0 \cdot 0001$; Fig. 1A). Overall, there were no differences between the groups in acquisition of the task during training trials (Fig. 1A) or in the accuracy of their searches during probe trials (Fig. 1B). At 18 months of age (Fig. 1C), a main effect of block indicated that all groups performed more accurately by the end of training and acquired the task $(F(3,3)=174 \cdot 63, P<0 \cdot 0001 ;$ Fig. 1 C $)$. A main effect
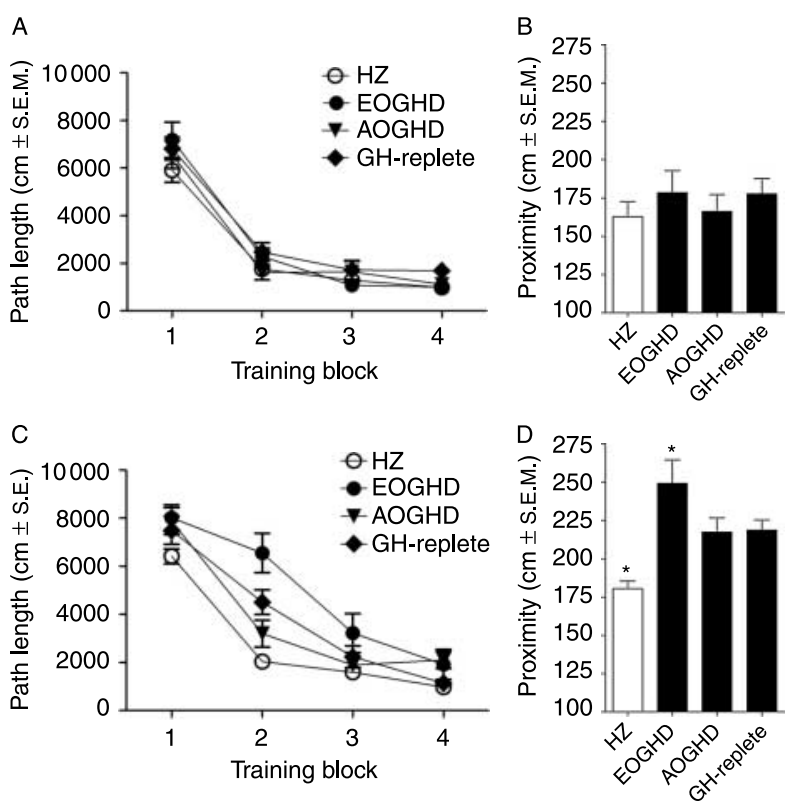

Figure 1 Spatial learning at 8 (A and $B$ ) and 18 months of age $(C$ and D) in heterozygous $(\mathrm{HZ})$ and dwarf rats. At 8 months of age, all groups showed similar performance in the acquisition of the escape platform location (A) and in probe trial performance (B). At 18 months of age, however, acquisition of the escape platform location (C) was significantly better in the $\mathrm{HZ}$ group compared to all other groups $(P<0 \cdot 05)$ and EOGHD rats were significantly impaired compared to all other groups, $P<0 \cdot 05$ (see text for further description). The search accuracy during the probe trial (D) was similarly affected, with the $\mathrm{HZ}$ group showing the best performance compared to all other groups $\left(P_{\mathrm{S}}<0 \cdot 05\right)$ as indicated by the lower proximity to the platform, and the EOGHD group showing the worst performance compared to all other groups. ${ }^{*} P<0 \cdot 05$ compared to all other groups. $\mathrm{HZ}$, heterozygous; EOGHD, early-onset $\mathrm{GH}$ deficiency; AOGHD, adult-onset GH deficiency.

of group $(F(3,41)=8 \cdot 88, P \leq 0 \cdot 0001)$, an interaction between group and block $(F(3,9)=3 \cdot 85, P<0 \cdot 0005)$ and a subsequent Fisher's PLSD post hoc, however, indicated that the HZ group performed significantly better than all other groups $(P<0 \cdot 05$, each) and that the EOGHD group performed significantly poorer than all other groups $(P<0 \cdot 05$, each; Fig. 1C). There were no significant differences in spatial learning between AOGHD or GH-replete groups indicating no further effect of continuing GH supplementation after the 10-week treatment period. Overall, the data indicate that by 18 months of age, AOGHD rats receiving GH supplementation from 4 to 14 weeks of age demonstrate cognitive performance that is better than that of EOGHD rats, but not fully improved to the level of the $\mathrm{HZ}$ group.

The probe trial data in Fig. 1D shows a similar pattern to the acquisition data described above: the HZ group had a significantly more accurate search for the escape platform compared to all other groups (main effect: $F(3,41)=10 \cdot 37$, $P<0 \cdot 0001$, post hoc, $P<0 \cdot 05)$ and the EOGHD group had the least accurate search in finding the location of the platform 
compared to all other groups (Fisher's PLSD post hoc, $P<0 \cdot 05)$. No significant differences in search accuracy were observed between AOGHD and GH-replete rats, indicating that additional GH supplementation after 14 weeks of age were not of added value in the prevention of spatial learning decline at 18 months of age.

Swim speed and visual ability were measured during the visible platform test (cue training). HZ and the dwarf groups were not different, nor was there an effect of GH treatment in the dwarfs at either 8 or 18 months of age (data not shown). These data indicate that the differences in spatial learning and memory ability are due to deficits in learning, and not due to group differences in sensory or motor ability.

\section{Plasma IGF1 levels and body weight}

IGF1 levels were measured in plasma obtained from tail blood 1 week after water maze testing. Figure 2 shows that at both 8 and 18 months of age, $\mathrm{HZ}$ and dwarf GH-replete rats had significantly higher plasma IGF1 levels compared to the EOGHD and AOGHD rats $(P \leq 0 \cdot 05$, each), indicating that twice daily administration of $\mathrm{GH}$ to the dwarf rats increases plasma IGF1 and that discontinuation of GH administration at 14 weeks of age results in a return to GH-deficiency (main effect of group, 8 months: $F(3,27)=56 \cdot 66, P<0 \cdot 0001$; 18 months: $F(3,39)=12 \cdot 73, P<0 \cdot 0001)$. At 8 months of age, the GH-replete group had slightly higher levels of IGF1 compared to the $\mathrm{HZ}$ group $(P \leq 0 \cdot 005)$, whereas at 18 months the GH-replete group had slightly lower levels than the $\mathrm{HZ}$ group $(P \leq 0 \cdot 05)$. These data could indicate an upregulation of some signaling mechanisms at 8 months of age that become less responsive by 18 months of age. There were no significant differences in IGF1 plasma levels between the AOGHD and EOGHD groups at either age. The $\mathrm{HZ}$ and GH-replete rats expressed the typical phenotype of adequate GH levels, indicated by increased body weight (Fig. 3). At both ages, the $\mathrm{HZ}$ and GH-replete rats weighed significantly more than the AOGHD and EOGHD groups $(8$ months; main effect
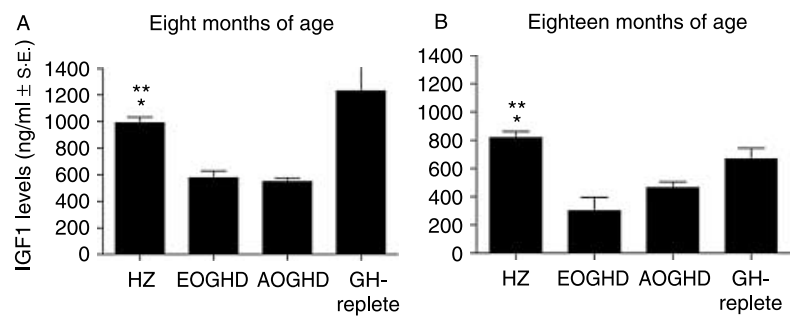

Figure 2 Plasma IGF1 levels after spatial learning assessment in 8 and 18 month-old heterozygous $(\mathrm{HZ})$ and dwarf rats. At both 8 and 18 months of age, plasma IGF1 levels were significantly higher in the $\mathrm{HZ}$ and $\mathrm{GH}$-replete groups compared to the EOGHD and AOGHD groups. EOGHD and AOGHD levels did not differ at either age. At 8 months of age, GH-replete rats had higher levels of IGF1 relative to all other groups, including the $\mathrm{HZ}$ rats $(P<0 \cdot 05)$. At 18 months of age, GH-replete rats had slightly lower levels of IGF1 compared to the $\mathrm{HZ}$ rats. ${ }^{*} P<0 \cdot 05$ from all other groups; ${ }^{* *} P<0 \cdot 05$ $\mathrm{HZ}$ versus $\mathrm{GH}$-replete.
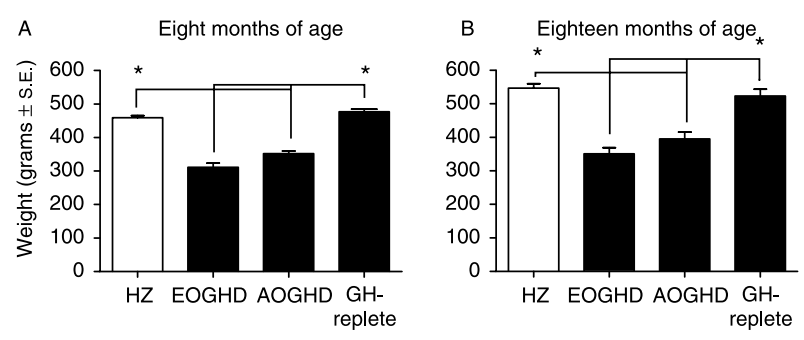

Figure 3 Body weight in 8 and 18 month-old heterozygous $(\mathrm{HZ})$ and dwarf rats. $\mathrm{GH}$-replete rats and $\mathrm{HZ}$ rats have comparable body weight but both have significantly greater weight than EOGHD and AOGHD rats. $* P<0 \cdot 05$.

$F(3,29)=94.50, \quad P<0 \cdot 0001 ; 18$ months; main effect $F(3,41)=21 \cdot 71, \quad P<0.0001$ and post hoc comparisons $P<0 \cdot 0001)$. Higher levels of serum IGF1 significantly correlated with higher body weight at both 8 and 18 months of age $(R=+0 \cdot 88, P<0 \cdot 0001$ and $R=+0 \cdot 64, P=0 \cdot 0001$, respectively). Overall, the data indicated that GH administration to dwarf rats significantly increased IGF1 plasma levels and body weights comparable to those in $\mathrm{HZ}$ rats.

\section{Discussion}

The data presented in the current study demonstrate that the absence of the rise of GH in dwarf rats between 4 and 14 weeks of age (EOGHD) results in spatial learning and memory impairment at 18 months of age compared to $\mathrm{HZ}$ rats. These results are in accordance with human studies that show a greater disturbance in cognitive function in EOGHD patients than in those that become GH deficient later in life (AOGHD) (van Dam et al. 2005). GH supplementation in dwarf rats during the 10 -week period to model AOGHD improved memory at 18 months of age, as indicated by the comparable spatial learning and memory ability between the $\mathrm{HZ}$ and AOGHD groups. Interestingly, the additional supplementation after 14 weeks of age (GH-replete) did not provide any additional therapeutic benefit. These data provide supportive evidence for the existence of an important period of the lifespan during which elevated levels of $\mathrm{GH}$ are necessary to delay or prevent cognitive impairment in mid-life.

The peripubertal rise in $\mathrm{GH}$, independent of $\mathrm{GH}$ levels in adulthood, may exert an unique effect on the brain and permanently alter brain neurochemistry and/or the neuronal circuitry necessary for the development and maintenance of neural circuitry in adulthood. This hypothesis is congruent with the clinical data indicating that continued $\mathrm{GH}$ administration throughout the transitional period between puberty and adulthood in COGHD patients may be necessary for normal somatic development manifest later in adulthood (Clayton et al. 2007, Nilsson et al. 2007, McCutcheon \& Marinelli 2009). In the human brain, diffusion tensor magnetic resonance imaging has shown continued maturation 
of certain brain connections, particularly fronto-temporal connections, into the second and third decades of life (Lebel et al. 2008). There is also evidence in humans and rodents that myelination and changes in dopaminergic neuron firing rate occur throughout the transitional adolescent period (Wiggins 1982, Hunter et al. 1997, Giedd et al. 1999). In the rat, there is evidence of continued brain maturation, measured by dopamine receptor levels, until postnatal day 60 (PND 60, 8 weeks of age) (Tarazi \& Baldessarini 2000), and some forms of hippocampal-dependent learning, such as spatial delayed alternation, are not evident until PND 40 (Stanton 2000). Thus, endocrine modulators such as GH and IGF1 reach peak levels just at precisely the time of formation and maintenance of critical connections. Dysfunctional regulation of brain development may be subtle and not fully apparent until revealed by the additional insult of increased age.

In humans, the effect of COGHD or AOGHD on cognition in adulthood generally has been associated with attentional and memory deficits along with mood disorders (Frankel \& Laron 1968, Laron \& Galatzer 1981, Galatzer et al. 1993, Falleti et al. 2006, van Nieuwpoort \& Drent 2008). For example, individuals with COGHD exhibit delayed verbal memory recall and deficits in the Trail Making Test A (a test of planning, processing speed and attention) and these deficits are associated with reductions in the brain $\mathrm{N}$-acetylaspartate/ choline ratio, a marker of neuronal integrity (van Dam et al. 2005). The optimal GH supplementation strategy to improve deficits in cognition in GHD patients is still highly debatable but there is some evidence that discontinuation of supplementation during the transitional adolescent period may impact somatic development (e.g. see Clayton et al. (2007)). GH substitution therapy does improve long-term and working memory in 27-year-old COGHD patients treated for 6 months (Arwert et al. 2006). Whether or not GH supplementation during the adolescent stage has greater impact than supplementation in adulthood is not known.

Previously published findings and the results reported in the present study provide compelling evidence that the administration of $\mathrm{GH}$ to deficient individuals in adolescence results in pleiotropic actions that are manifest late during the lifespan. For example, replacement of GH to dwarf rats only from postnatal week 4 through week 14 produces AOGHD and increases lifespan by 15\% (Sonntag et al. 2005). This relatively circumscribed exposure to $\mathrm{GH}$ during postnatal weeks 4-14 also appears to influence the timing of intracerebral hemorrhage or cardiac thrombus in the older brain (Sonntag et al. 2005). These results have led to the conclusion that manipulation of $\mathrm{GH}$ for a relatively brief period during the transitional period between puberty and young adulthood is sufficient to alter age-related pathology later in life, although future studies are needed to identify the explicit boundaries of the optimal treatment time. The present data add to our understanding of this important period of brain growth by indicating that the absence of GH during this time period is also a risk factor for the early emergence of cognitive deficits at middle age.
In conclusion, our results indicate that GHD in adolescence deleteriously impacts brain function later in life and may result in the early emergence of age-related deficiencies in learning and memory. Supplementation of dwarf rats with GH during this period results in performance comparable to that of $\mathrm{HZ}$ rats and additional GH supplementation after 14 weeks of age has no additional benefits. The specific mechanisms for the effects of GH during the transitional period remain to be determined, but may include the formation or stabilization of the microcircuitry of essential synaptic transmission (Ramsey et al. 2005, Mahmoud \& Grover 2006).

\section{Declaration of interest}

There is no conflict of interest that could be perceived as prejudicing the impartiality of the research reported.

\section{Funding}

Funding was provided by the National Institute of Aging P01 AG011370 to WES, MMN, and ENM and R01 AG26607 to WES.

\section{Acknowledgements}

The authors greatly appreciate the expert technical assistance of Constance Linville and Mona Beardslee.

\section{References}

Adams MM, Elizabeth Forbes M, Constance Linville M, Riddle DR, Sonntag WE \& Brunso-Bechtold JK 2009 Stability of local brain levels of insulinlike growth factor-I in two well-characterized models of decreased plasma IGF-I. Growth Factors 27 181-188.

Adem A, Jossan SS, d'Argy R, Gillberg PG, Nordberg A, Winblad B \& Sara V 1989 Insulin-like growth factor 1 (IGF-1) receptors in the human brain: quantitative autoradiographic localization. Brain Research $\mathbf{5 0 3}$ 299-303.

Arwert LI, Veltman DJ, Deijen JB, van Dam PS \& Drent ML 2006 Effects of growth hormone substitution therapy on cognitive functioning in growth hormone deficient patients: a functional MRI study. Neuroendocrinology 83 12-19.

Carter CS, Ramsey MM, Ingram RL, Cashion AB, Cefalu WT, Wang ZQ \& Sonntag WE 2002a Models of growth hormone and IGF-1 deficiency: applications to studies of aging processes and life-span determination. Journals of Gerontology. Series A, Biological Sciences and Medical Sciences $\mathbf{5 7}$ B177-B188.

Carter CS, Ramsey MM \& Sonntag WE $2002 b$ A critical analysis of the role of growth hormone and IGF-1 in aging and lifespan. Trends in Genetics $\mathbf{1 8}$ 295-301.

Charlton HM, Clark RG, Robinson IC, Goff AE, Cox BS, Bugnon C \& Bloch BA 1988 Growth hormone-deficient dwarfism in the rat: a new mutation. Journal of Endocrinology 119 51-58.

Clayton P, Gleeson H, Monson J, Popovic V, Shalet SM \& Christiansen JS 2007 Growth hormone replacement throughout life: Insights into age-related responses to treatment. Growth Hormone \& IGF Research 17 369-382. 
van Dam SP, de Winter CF, de Vries R, van der Grond J, Drent ML, Lijffijt M, Leon Kenemans J, Aleman A, de Haan EHF \& Koppeschaar HPF 2005

Childhood-onset growth hormone deficiency, cognitive function and brain $N$-acetylaspartate. Psychoneuroendocrinology 30 357-363.

Falleti MG, Maruff P, Burman P \& Harris A 2006 The effects of growth hormone $(\mathrm{GH})$ deficiency and $\mathrm{GH}$ replacement on cognitive performance in adults: a meta-analysis of the current literature. Psychoneuroendocrinology 31 681-691.

Frankel JJ \& Laron Z 1968 Psychological aspects of pituitary insufficiency in children and adolescents with special reference to growth hormone. Israel Journal of Medical Sciences 4 953-961.

Galatzer A, Aran O, Nagelberg N, Rubitzek J \& Laron Z 1993 Cognitive and psychosocial functioning of young adults with Laron syndrome. Pediatric and Adolescent Endocrinology 24 53-60.

Gallagher M, Burwell R \& Burchinal M 1993 Severity of spatial learning impairment in aging: development of a learning index for performance in the Morris water maze. Behavioral Neuroscience 107 618-626.

Gallagher M, Bizon JL, Hoyt EC, Helm KA \& Lund PK 2003 Effects of aging on the hippocampal formation in a naturally occurring animal model of mild cognitive impairment. Experimental Gerontology 38 71-77.

Giedd JN, Blumenthal J, Jeffries NO, Castellanos FX, Liu H, Zijdenbos A, Paus T, Evans AC \& Rapoport JL 1999 Brain development during childhood and adolescence: a longitudinal MRI study. Nature Neuroscience $\mathbf{2}$ 861-863.

Hunter SF, Leavitt JA \& Rodriguez M 1997 Direct observation of myelination in vivo in the mature human central nervous system. A model for the behaviour of oligodendrocyte progenitors and their progeny. Brain 120 2071-2082.

Lai Z, Roos P, Zhai O, Olsson Y, Fholenhag K, Larsson C \& Nyberg F 1993 Age-related reduction of human growth hormone-binding sites in the human brain. Brain Research 621 260-266.

Laron Z \& Galatzer A 1981 Effect of hGH on head circumference and IQ in isolated growth hormone deficiency. Early Human Development 5 211-214.

LaSarge CL \& Nicolle MM 2009 Comparison of different cognitive rat models of human aging. In Animal Models of Human Cognitive Aging, pp 73-102. Eds JL Bizon \& AG Wood. New York: Humana Press.

Lebel C, Walker L, Leemans A, Phillips L \& Beaulieu C 2008 Microstructural maturation of the human brain from childhood to adulthood. NeuroImage 40 1044-1055.

Mahmoud GS \& Grover LM 2006 Growth hormone enhances excitatory synaptic transmission in area CA1 of rat hippocampus. Journal of Neurophysiology 95 2962-2974.

Markowska AL, Mooney M \& Sonntag WE 1998 Insulin-like growth factor-1 ameliorates age-related behavioral deficits. Neuroscience 87559.
McCutcheon JE \& Marinelli M 2009 Age matters. European Journal of Neuroscience 29 997-1014.

van Nieuwpoort IC \& Drent ML 2008 Cognition in the adult with childhood-onset GH deficiency. European Journal of Endocrinology 159 S53-S57.

Nilsson AG, Svensson J \& Johannsson G 2007 Management of growth hormone deficiency in adults. Growth Hormone \& IGF Research 17 441-462.

Nyberg F 2000 Growth hormone in the brain: characteristics of specific brain targets for the hormone and their functional significance. Frontiers in Neuroendocrinology 21 330-348.

Ramsey MM, Adams MM, Ariwodola OJ, Sonntag WE \& Weiner JL 2005 Functional characterization of des-IGF-1 action at excitatory synapses in the CA1 region of rat hippocampus. Journal of Neurophysiology 94 247-254.

Rice D \& Barone S Jr 2000 Critical periods of vulnerability for the developing nervous system: evidence from humans and animal models. Environmental Health Perspectives 108 511-533.

Sonntag WE, Lynch CD, Bennett SA, Khan AS, Thornton PL, Cooney PT, Ingram RL, McShane T \& Brunso-Bechtold JK 1999 Alterations in insulin-like growth factor-1 gene and protein expression and type 1 insulin-like growth factor receptors in the brains of ageing rats. Neuroscience 88 269-279.

Sonntag WE, Carter CS, Ikeno Y, Ekenstedt K, Carlson CS, Loeser RF, Chakrabarty S, Lee S, Bennett C, Ingram R et al. 2005 Adult-onset growth hormone and insulin-like growth factor I deficiency reduces neoplastic disease, modifies age-related pathology, and increases life span. Endocrinology $1462920-2932$.

Stanton ME 2000 Multiple memory systems, development and conditioning. Behavioural Brain Research 110 25-37.

Tarazi FI \& Baldessarini RJ 2000 Comparative postnatal development of dopamine D1, D2 and D4 receptors in rat forebrain. International Journal of Developmental Neuroscience 18 29-37.

Wiggins RC 1982 Myelin development and nutritional insufficiency. Brain Research 257 151-175.

Received in final form 2 October 2009

Accepted 8 October 2009

Made available online as an Accepted Preprint

8 October 2009 